\title{
Holographic non-equilibrium heating
}

\section{D.S. Ageev and I.Ya. Aref'eva}

Steklov Mathematical Institute, Russian Academy of Sciences, Gubkina str. 8, 119991, Moscow, Russia

E-mail: ageev@mi.ras.ru, arefeva@mi.ras.ru

ABSTRACT: We study the holographic entanglement entropy evolution after a global sharp quench of thermal state. After the quench, the system comes to equilibrium and the temperature increases from $T_{i}$ to $T_{f}$. Holographic dual of this process is provided by an injection of a thin shell of matter in the black hole background. The quantitative characteristics of the evolution depend substantially on the size of the initial black hole. We show that characteristic regimes during non-equilibrium heating do not depend on the initial temperature and are the same as in thermalization. Namely these regimes are pre-localequilibration quadratic growth, linear growth and saturation regimes of the time evolution of the holographic entanglement entropy. We study the initial temperature dependence of quantitative characteristics of these regimes and find that the critical exponents do not depend on the temperature, meanwhile the prefactors are the functions on the temperature.

KEYWORDS: AdS-CFT Correspondence, Holography and condensed matter physics (AdS/CMT)

ARXIV EPRINT: 1704.07747 


\section{Contents}

1 Introduction 1

2 Holographic entanglement entropy in dBTZ-Vaidya background 2

2.1 The dual geometry 2

2.2 The geodesic length 3

2.3 Critical curve 5

3 Universality of critical behaviour $\quad 7$

$\begin{array}{lll}3.1 & \text { Regimes in holographic heating } & 7\end{array}$

$\begin{array}{lll}3.2 & \text { Pre-local equilibration growth } & 7\end{array}$

3.3 Memory loss regime $\quad 9$

3.3.1 Post-local-equilibration linear growth. Expansion near $\vartheta_{\kappa} \quad 10$

$\begin{array}{lll}3.3 .2 & \text { Saturation. Expansion near } \pi / 2 & 11\end{array}$

3.3.3 Late-time memory loss regime. Interpolation between $\phi=\vartheta_{\kappa}$ and $\pi / 212$

4 Conclusions and discussions

\section{Introduction}

It is universally recognized that one of the most challenging problems in quantum theory is the description of the equilibration process, in particular, the thermalization. The AdS/CFT correspondence [1-3] proposes a powerful tool for a description of thermalization process in general class of strongly coupling quantum theories. In the holographic duality, the temperature in the quantum field theory is related with the black hole (or black brane) temperature in the dual background [4]-[6]. The thermalization within AdS/CFT duality corresponds to a black hole formation, see [7-11]. The simplest description of a black hole formation process is provided by the Vaidya deformation of a given background. This model serves as a relatively simple and universal holographic model of thermalization with a wide range of applicability in different physical situations, see [13]-[51] and references therein. Thick shell Vaidya models can be solved only numerically, meanwhile several thin shell models admit an analytical solution $[13,14,16]$. Thin shell models capture all features of evolution during sharp quenches $[19,20]$ and in the low-dimensional case reproduce results obtained in the conformal field theory $[52,53]$.

All these models have zero initial temperature. However, not all interesting nonequilibrium processes in strongly correlated systems start from zero temperature. Especially, this concerns phenomena related to biological systems. In particular, application of holographic approach to a part of the photosynthetic process [45] requires consideration of the global quench of thermal states. From non-holographic point of view, a global 
quench in quantum field theory starting from a thermal initial state has been considered for massive non-interacting field models in [54].

In this paper, in the holographic approach, we study the simplest process associated with a global quench of the thermal initial state, which leads to an increase in temperature. As a holographic dual to this process, we use the so-called double-BTZ-Vaidya background. This background interpolates between two AdS black holes with different temperatures. The thin shell limit of this background corresponds to the sharp quench. We show that holographic non-equilibrium heating inherits typical properties of holographic thermalization. Behaviour of the holographic entanglement entropy (HEE) during thermalization have been studied in details by H.Liu and J.Suh [19, 20], see also [17, 22, 49]. They have shown, that during thermalization the HEE passes several regimes, namely, the pre-local-equilibration quadratic growth, the post-local-equilibration linear growth, the late-time regime, and the saturation regime. We show that all these regimes are present in non-equilibrium heating. We also show that although the evolution of the large regions of entanglement entropy is controlled by the geometry around and within the horizon of the emerging black hole, the quantitative characteristics of evolution from the thermal state also depend substantially on the horizon of the initial black hole. We calculate the corrections to different scaling coefficients taking into account non-zero initial temperature.

The paper is organized as follows. In section 2.1 we sketch the dual geometry of nonequilibrium heating process. Then in section 2.2 we present the new explicit formulae for the entanglement entropy evolution after the global quench of initially thermal state. After that, in section 2.3, we study in details relations between bulk characteristics of the geodesics anchored on the interval and the boundary data. In section 3.1 we list the specific regimes in non-equlibrium heating and compare with some previous results about behavior of the entanglement entropy during thermalization. Section 3.2 is devoted to the initial quadratic growth regime. In section 3.3 we compute the scaling characteristics of the memory loss regime in the process of the global quench of thermal state with temperature $T_{i} \neq 0$. We find out the universal critical behaviour of the scaling exponents for $T_{i} \neq=0$. The main focus here is on their dependences on the final and initial temperatures. In the final section 4 we conclude and we discuss some possible generalizations.

\section{Holographic entanglement entropy in dBTZ-Vaidya background}

\subsection{The dual geometry}

We are interested in the evolution of holographic entanglement entropy of the interval of length $\ell$ and at time $\tau$ in holographic setup described by the double-BTZ-Vaidya quench (see figure 1). We call double-BTZ-Vaidya or dBTZ-Vaidya the geometry that describes shell of null matter accreting in the black hole background. We focus on the thin shell limit of this metric. In contrast to usual BTZ-Vaidya geometry (sometimes it is called AdS-Vaidya geometry) black hole is present from the very beginning of process. Thus we consider black hole evolving from the initial state defined by the horizon position $z_{H}$ to the final state with horizon $z_{h}$ as a dual background. The dBTZ-Vaidya metric in the thin 


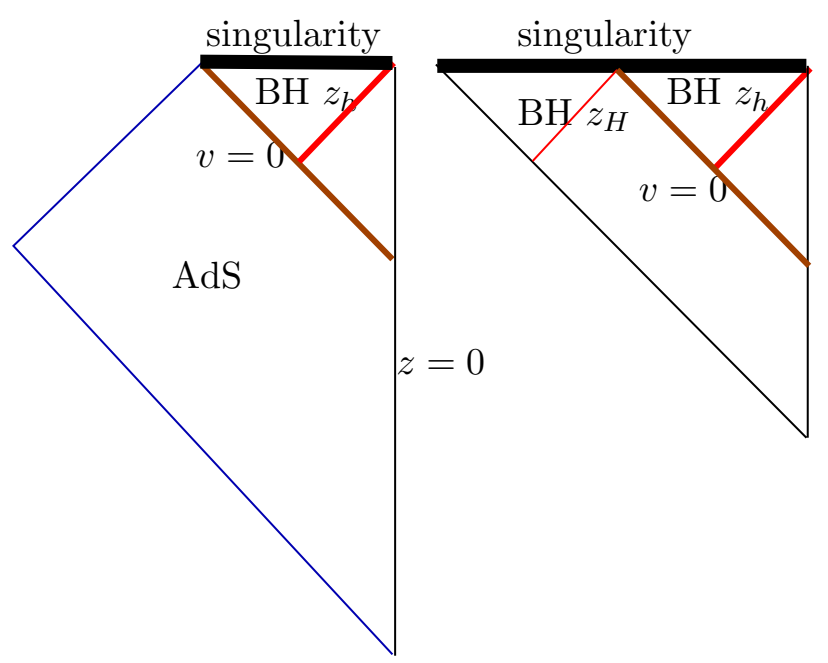

Figure 1. Penrose diagrams for double-BTZ-Vaidya geometry defined by (2.1) and (2.2). The left plot corresponds to $z_{H}=0$ (usual BTZ-Vaidya spacetime), the right plot corresponds to the case $z_{H}>0$.

shell limit is given by

$$
d s^{2}=\frac{R^{2}}{z^{2}}\left(-f(z, v) d v^{2}-2 d v d z+d \vec{x}^{2}\right), \quad f(z, v)=\theta(v) f_{h}(z)+\theta(-v) f_{H}(z)
$$

and functions $f_{H}$ and $f_{h}$ are defined as

$$
f_{H}=1-\left(\frac{z}{z_{H}}\right)^{2}, \quad f_{h}=1-\left(\frac{z}{z_{h}}\right)^{2}, \quad z_{h}<z_{H},
$$

where the time is related with variable $v$ as

$$
v<0: \quad t=v+z_{H} \operatorname{arctanh} \frac{z}{z_{H}} ; \quad v>0: \quad t=v+z_{h} \operatorname{arctanh} \frac{z}{z_{h}} .
$$

Usual holographic BTZ-Vaidya setup is recovered in the limit $z_{H} \rightarrow \infty$. The initial and final temperatures and energy densities are

$$
T_{i}=\frac{1}{2 \pi z_{H}}, \quad T_{f}=\frac{1}{2 \pi z_{h}}, \quad \varepsilon_{i}=\frac{R}{8 \pi G_{N}} \frac{1}{z_{H}^{2}}, \quad \varepsilon_{f}=\frac{R}{8 \pi G_{N}} \frac{1}{z_{h}^{2}},
$$

where $G_{N}$ is Newton's constant in the bulk and $R$ is a typical scale in the bulk. Note, that the case $z_{H}<z_{h}$ corresponds to a model of cooling [15] and this model violates NEC condition [19].

\section{$2.2 \quad$ The geodesic length}

Now let us turn to the description of the holographic entanglement entropy of the interval in non-equilibrium heating process dual to the metric (2.1). This entanglement 
entropy is obtained explicitly as a length of the geodesic that are spacelike, with both endpoints anchored on the boundary at the same time $\tau$ and length separation $\ell$. Also these geodesics are called ETEBA (Equal-Time Endpoints Boundary Anchored) geodesics for brevity, see [24]. In this paper we set $R=1$ and also ignore the factors of $4 G$ identifying the length of ETEBA geodesics with the entanglement entropy. This class of geodesics in dBTZ geometry has been considered in [23]. For the sake of convenience we introduce parameter $\kappa=z_{h} / z_{H}$. The formula for entanglement entropy has the form

$$
S=\log \left(\frac{R}{\varepsilon} \frac{z_{h}}{\ell \mathfrak{S}_{\kappa}(\rho, s)} \sinh \frac{\tau}{z_{h}}\right),
$$

where the form of $\mathfrak{S}_{\kappa}$ will be given below (see formula (2.7)). This formula has the form similar to that from [26] and in the limit $\kappa \rightarrow 0$ the function $\mathfrak{S}_{0}(\rho, s)=s$ recovering the result of [26]. Formula (2.5) contains parameters $\rho$ and $s$ related with the bulk characteristics of the geodesic

$$
s=\frac{z_{c}}{z_{*}}, \rho=\frac{z_{h}}{z_{c}}, c=\sqrt{1-s^{2}}, s=\sin \phi,
$$

where $z_{c}$ is the point where the geodesic crosses the shell and $z_{*}$ is the turning point of the geodesic. Using these parameters the explicit formula for $\mathfrak{S}_{\kappa}$ has the form

$$
\mathfrak{S}_{\kappa}(\rho, s)=\frac{c \rho+\Delta}{\Delta} \cdot \sqrt{\frac{\Delta^{2}-c^{2} \rho^{2}}{\rho\left(c^{2} \rho+2 c \Delta+\rho\right)-\kappa^{2}}},
$$

and for simplicity we define $\gamma=1-\kappa^{2}$ and $\Delta=\sqrt{\rho^{2}-\kappa^{2}}$. The final ingredient to describe the geodesic length is the relation obtained in [23] between boundary separation $\ell$, time $\tau$ and bulk data $\rho$ and $s$. These formulae, being quite complicated are the explicit generalization of the similar relation from [26]. Let us define the total length $\ell$ as $\ell=$ $\ell_{-}+\ell_{+}$. Here $\ell_{-}$is the length of the part of the geodesic under the shell, i.e. between points $z_{*}$ and $z_{c}$, while $\ell_{-}$is the length of the part of the geodesic connecting points $z_{c}$ and boundary $z=0$ at $x=\ell$ over the shell. The expressions for $\tau$ and $\ell$ take the form

$$
\begin{aligned}
\frac{\tau}{z_{h}} & =\operatorname{arccoth}\left(\frac{-c \kappa^{2}+2 c \rho^{2}+c+2 \Delta \rho}{2 c \rho+2 \Delta}\right), \\
\ell_{+} & =\frac{z_{h}}{2} \log \left(\frac{c^{2} \gamma^{4}-4 \Delta\left(c s\left(\kappa^{2}-2 \rho^{2}+1\right)+\Delta+\Delta\left(\rho^{2}-2\right) s^{2}\right)}{c^{2} \gamma^{4}-4 \Delta^{2}(\rho s-1)^{2}}\right), \\
\ell_{-} & =\frac{z_{h}}{2 \kappa} \log \left(\frac{(c \kappa+\Delta s)^{2}}{\rho^{2} s^{2}-\kappa^{2}}\right) .
\end{aligned}
$$

It is useful to consider the difference $\Delta S$ between the entanglement at the current time moment $S(\ell, \tau)$ and the final state entanglement entropy value $S_{\text {eq }}$

$$
\Delta S(\ell, \tau)=S(\ell, \tau)-S_{\text {eq }}(\ell),
$$

where $S_{\text {eq }}$ is defined as

$$
S_{\text {eq }}=\log \left(\frac{R}{\varepsilon} \frac{z_{h}}{\ell} \sinh \frac{\ell}{z_{h}}\right) .
$$


The bulk variables define relative position of the geodesic top $z_{*}$ and the point where geodesic crosses the null shell $z_{c}$ with restrictions $z_{*}<z_{H}$ and $z_{c}<z_{*}$. There are still 3 types of ETEBA geodesics as in [13, 19, 20].

For $\tau<0$ our ETEBA geodesic (first type) lies entirely in the BTZ bulk with temperature $T_{i}$. The entanglement entropy in this case is independent of $\tau$ and equals to

$$
S_{i}=\log \left(\frac{R}{\varepsilon} \frac{z_{H}}{\ell} \sinh \frac{\ell}{z_{H}}\right),
$$

where $\varepsilon$ is the UV regularization. For $\tau<0$ the limit of large interval length $\ell \rightarrow \infty$ corresponds to $z_{*} \rightarrow z_{H}$ (here we mean that $z_{*} \rightarrow z_{H}-0$ ) in accordance with [49]. Let us fix $\ell$ and start to increase the time $\tau$.

At very small $\tau>0$, the ETEBA geodesic starts intersecting the null shell and for $\tau \ll z_{h}$ the point of intersection is close to the boundary, $z_{c} \ll z_{h}$. This is the second type of the ETEBA geodesics.

When $\tau$ is of order $z_{h}$ the ETEBA geodesic (third type) intersects the shell behind the horizon, i.e. $z_{c}>z_{h}$. At some time $\tau=\tau_{s}$ the ETEBA geodesic lies entirely in the black hole (with the temperature $T_{f}$ ) region. The role of the second horizon located at $z=z_{H}$ is that it pulls out of the ETEBA geodesic with the top $z_{*}>z_{h}$ to the first horizon, and this effect is stronger as the difference of two temperatures decrease, i.e. $\kappa \rightarrow 1$.

The described ETEBA geodesics length also is related to the equal-time two-point correlation function. The equal-time two-point function $\left\langle\mathcal{O}(x, t) \mathcal{O}\left(x^{\prime}, t\right)\right\rangle$ of operators $\mathcal{O}$ with large conformal dimension $\Delta$ between points on the boundary $x$ and $x^{\prime}$ at a time moment $t$ is expressed as

$$
G\left(x, x^{\prime}, t\right)=e^{-\Delta \mathcal{L}\left(x, x^{\prime}, t\right)},
$$

where $\mathcal{L}\left(x, x^{\prime}, t\right)$ is the corresponding geodesic length. The geodesics described above are responsible for different regimes of behavior of the two-point correlator.

\subsection{Critical curve}

Formula (2.8) gives the explicit dependence for $\ell$ and $\tau$ from the geometry of the entanglement surface specified by parameters $\rho$ and $\phi$. For any $0 \leq \kappa \leq 1$ there is a critical curve in the parameter space $\rho$ and $\phi$, so that only on the right of this line (the red line in figure 2) one can perform the single-valued change of variables $(\rho, \phi) \rightarrow(\ell, \tau)$. To visualize this change of variables it is convenient to draw the lines of fixed values of $\ell$ and $\tau$ (brown and blue lines in figure 2). From figure 2 we see that large values of $\ell$ and $\tau$ are located near the critical line. The explicit form of the critical line $\rho=\rho_{*}(\phi)$ is defined by the function $\rho_{*}=\frac{1}{2} \mathcal{V}(\kappa, \phi)$,

$$
\mathcal{V}(\kappa, \phi) \equiv 1+(1-\mathcal{Q}) \csc \phi, \quad \mathcal{Q}(\kappa, \phi) \equiv \sqrt{\left(1-2 \kappa^{2}\right) \cos ^{2} \phi+2 \kappa^{2}(1-\sin \phi)} .
$$

Substituting $\rho_{*}$ into (2.8) one can check, that $\tau$ and $\ell_{+}$are equal to infinity on the critical line. Near the critical line we have

$$
\left.\frac{-c \kappa^{2}+2 c \rho^{2}+c+2 \rho \sqrt{\rho^{2}-\kappa^{2}}}{2 c \rho+2 \sqrt{\rho^{2}-\kappa^{2}}}\right|_{\rho=\rho^{*}+\epsilon}=1+2 \epsilon \mathcal{K}_{\tau}(\phi, \kappa)+\mathcal{O}\left(\epsilon^{2}\right),
$$




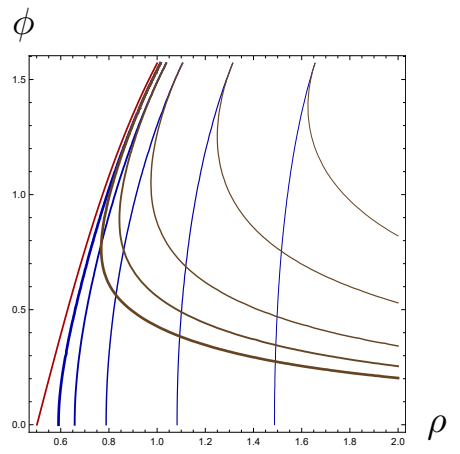

$\kappa=0$

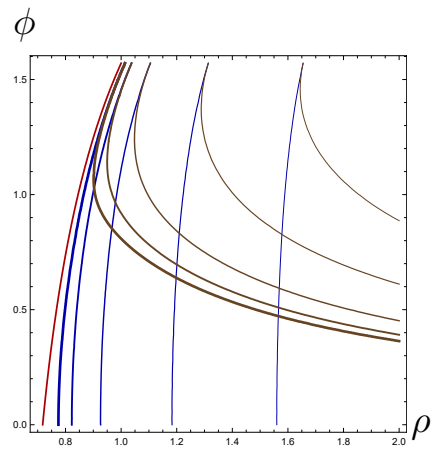

$\kappa=0.667$

Figure 2. The contour plots for $\ell$ and $\tau$ as functions of $\rho$ and $\phi$ for different $\kappa=0$ and $\kappa=0.667$. These plots show that on the right of the critical line (the darker red line) for $\ell>\tau$ we can always find solution to the system of equations: $\tau=\tau(\rho, \phi) ; \ell=\ell(\rho, \phi)$. Lines of the equal times $\tau=0.7,1,1.5,2,2.5$ in all panels are shown by blue color with increasing thickness from right to left. Lines of the equal $\ell=0.7,1,1.5,2,2.5$ in all panels are shown by brown with increasing thickness from up to bottom.

where

$$
\mathcal{K}(\kappa, \phi)=\left.\frac{c^{2} \Delta\left(\kappa^{2}+2 \rho^{2}-1\right)+c \rho\left(-3 \kappa^{2}+4 \rho^{2}-1\right)+2 \Delta^{3}}{4 \Delta(c \rho+\Delta)^{2}}\right|_{\rho=\rho_{*}},
$$

and, therefore, we get the following time asymptotic near the critical curve

$$
\tau=-\frac{z_{h}}{2} \log \varepsilon-\frac{z_{h}}{2} \log \mathcal{K}+\mathcal{O}(\varepsilon) .
$$

The expansion for $\ell_{+}$takes the similar form

$$
\ell_{+} \approx-\frac{z_{h}}{2} \log \epsilon+\frac{z_{h}}{2} \log \mathcal{K}^{+},
$$

where the function $\mathcal{K}^{+}=\frac{\mathfrak{N}}{\mathfrak{\kappa}}$ is defined as

$$
\begin{aligned}
\mathfrak{K}= & 2 \sqrt{2} Q \sin \phi-4 \cos ^{2} \phi+4 \kappa^{2}(2 \sin \phi(1-\sin \phi)-Q), \\
\mathfrak{N}= & 4\left(\kappa^{2}-1\right)^{2} \cos ^{2} \phi-\frac{\left(\mathcal{V}^{2}-4 \kappa^{2}\right)\left(\left(\mathcal{V}^{2}-8\right) \sin ^{2} \phi+4\right)}{4}+ \\
& +\frac{1}{2} \sqrt{\mathcal{V}^{2}-4 \kappa^{2}}\left(\mathcal{V}^{2}-2\left(\kappa^{2}+1\right)\right) \sin (2 \phi),
\end{aligned}
$$

where $\mathcal{V}$ and $Q$ is defined by (2.14).

The expression for $\ell_{-}$is not singular on the critical line

$$
\ell_{-}=\frac{z_{h}}{2 \kappa} \log \mathcal{K}^{-}, \quad \mathcal{K}^{-}=\frac{2 \sin \phi \sqrt{\mathcal{V}^{2}-4 \kappa^{2}}+4 \kappa \cos \phi}{\mathcal{V}^{2} \sin ^{2} \phi-4 \kappa^{2}} .
$$

In the limit of $\kappa \rightarrow 0$ we reproduce the expressions from [19]

$$
\mathcal{K}(\kappa, \phi) \underset{\kappa \rightarrow 0}{\rightarrow} 1+\cot \frac{\phi}{2}, \quad \mathcal{K}^{+}(\kappa, \phi) \underset{\kappa \rightarrow 0}{\rightarrow} \frac{\cot \frac{\phi}{2}+1}{\cot \frac{\phi}{2}}, \quad \mathcal{K}^{-}(\kappa, \phi) \underset{\kappa \sim 0}{\approx} 1 .
$$




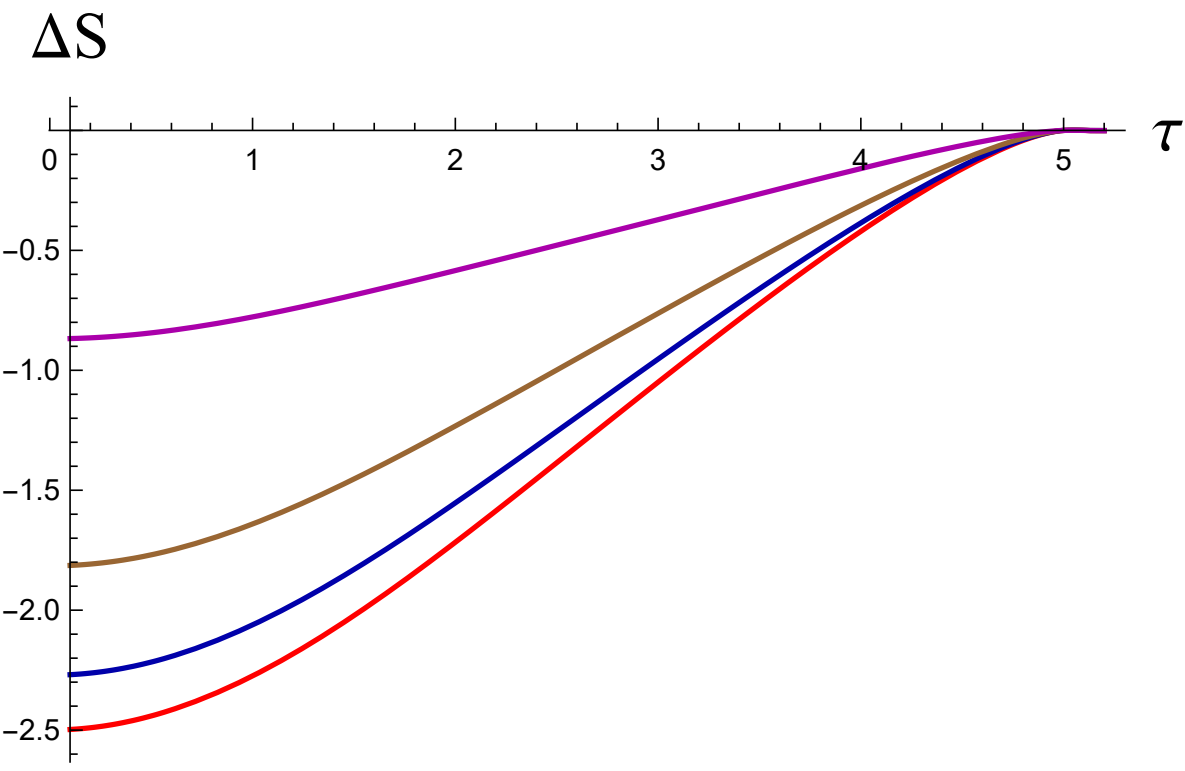

Figure 3. The dependence of $\Delta S$ on time $\tau$ for $\ell=5$ for different values of $z_{H}=\infty, 3,2,1.3$ from down to top.

\section{Universality of critical behaviour}

\subsection{Regimes in holographic heating}

In this subsection we establish different regimes of heating using the asymptotic behavior of our explicit formula (2.5). The regimes classification and notion of memory loss for BTZVaidya model have been considered in [19]. The equilibration starting from initial thermal state shares all basic regimes of thermalization process. There are the following regimes corresponding to different asymptotics for $\Delta S(\ell, \tau)=S-S_{\text {eq }}$. For time dependence of the entanglement entropy corresponding to different initial temperatures see figure 3 . Also see figure 4 below where we plot the comparison for approximations of different regimes asymptotics on timescale versus exact formula. It is worth to note a smooth character of the saturation regime (magenta and red lines in figure 4).

Namely

- Pre-local-equilibration growth regime is considered in 3.2

- Memory loss and subregimes of this regime is the main subject of section 3.3. Postlocal-equilibration linear growth is considered in 3.3.1, the saturation subregime is the subject of subsection 3.3.2 and late-time memory loss is briefly discussed in 3.3.3.

\subsection{Pre-local equilibration growth}

In [19] it was found, that the entanglement entropy in the system with zero initial temperature following the sharp quench first of all exhibits the period of quadratic growth. As in zero initial temperature case the quadratic growth regime is also present for $T_{i}>0$. 


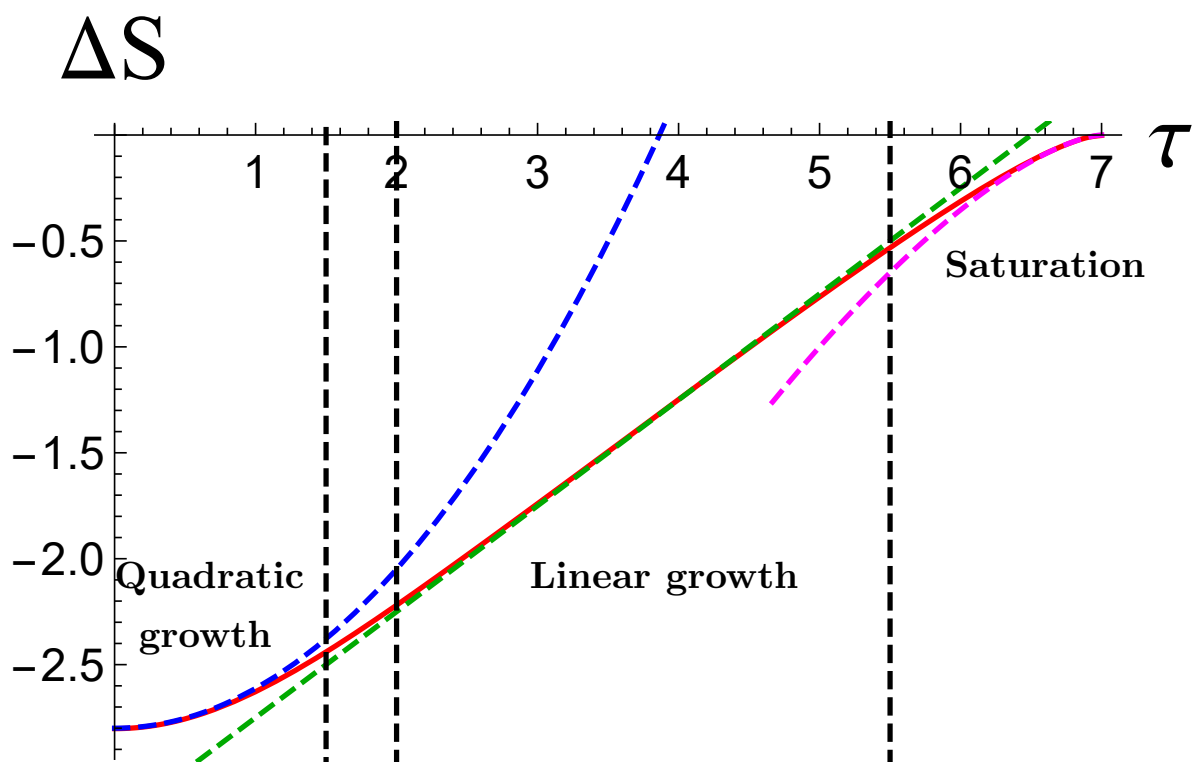

Figure 4. Typical time dependence of $\Delta S(\ell, \tau)$ for some fixed $\ell$ and $z_{H}$ (here $\ell=7$ and $z_{H}=2$ ). The red curve is the entanglement entropy dependence $\Delta S(\ell, \tau)$, the blue curve is the quadratic approximation to initial growth (3.3), the green line is the linear growth regime (3.18) and magenta line corresponds to the asymptotic describing saturation regime (3.24).

This regime takes place before the local equilibration defined by the scale $\xi \approx 1 / T_{f}$ is established. Pre-local equilibration growth regime occurs for small $\tau$, namely when $\tau \ll z_{h}$. Here we consider this regime assuming that $\ell \rightarrow 0$.

Using equations (2.8) when $\phi \rightarrow 0$ and $\tau / z_{h} \rightarrow 0$ we get the asymptotic for $\rho$ corresponding to this limit

$$
\rho \approx \frac{z_{h}}{\tau}+B \frac{\tau}{z_{h}}, \quad B=\frac{3 \kappa^{2}+1}{12} .
$$

Also from (2.8) we get the expansion for $\ell$ in the form

$$
\ell \approx \frac{t}{\sin \phi}+\frac{\kappa^{2} t^{3}}{3 z_{h}^{2} \sin ^{3} \phi}-\frac{3 \kappa^{2}+1}{12} \frac{t^{3}}{z_{h}^{2} \sin \phi} .
$$

From equation (2.5), and expansions (3.1) and (3.2) we get that $\Delta S$ (in the limit $\ell \rightarrow 0$ ) has the form

$$
\begin{aligned}
\Delta S & \approx \mathfrak{g}_{\kappa} \cdot \tau^{2}, \\
\mathfrak{g}_{\kappa} & =\frac{\left(1-\kappa^{2}\right)}{4 z_{h}^{2}} .
\end{aligned}
$$

This is the behavior of entanglement entropy in pre-local equilibration growth regime also known as quadratic growth regime. We explicitly observe the dependence on initial temperature. One can rewrite (3.3) using (2.4) in the form

$$
\Delta S \approx 2 \pi\left(\mathcal{E}_{f}-\mathcal{E}_{i}\right) \tau^{2} .
$$


Similar to the thermalization case before the local equilibration of the system the data characterizing the initial growth is the energy density difference between initial and final state.

\subsection{Memory loss regime}

Memory loss and subregimes of this regime [19] are the main subjects of this section. This regime occurs when both $\tau$ and $\ell$ are larger than $z_{h}$

$$
z_{h} \ll \tau \leq \ell
$$

Memory loss means that after the time when local equilibrium is established the entanglement propagation has the dependence only on difference $\ell-\tau$ instead of $\ell$ and $\tau$ separately. Depending on the ratio of $\ell-t$ to $\ell, \tau, z_{h}$ there are the following more detailed subregimes cases. The first subregime we discuss is post-local-equilibration linear growth, with entropy dependence

$$
\Delta S \approx(1-\kappa)(\ell-\tau)
$$

where $\ell$ is large and $z_{h} \ll \tau \ll \ell$. Then we consider the saturation regime, characterized by the entanglement entropy dependence

$$
\Delta S \approx-\mathfrak{f}_{\kappa}\left(\frac{\ell-\tau}{z_{h}}\right)^{3 / 2}-\mathfrak{n}_{\kappa}\left(\frac{\ell-\tau}{z_{h}}\right)^{2}
$$

where coefficients $\mathfrak{f}_{\kappa}$ and $\mathfrak{n}_{\kappa}$ depend on the initial temperature. These coefficients are calculated in section 3.3.2. The validity of this regime is provided when $\ell-\tau \ll z_{h}$. The last regime is the late-time memory loss regime. It occurs when $\ell \gg \ell-\tau \gg z_{h}$. We briefly discuss this regime in section 3.3.3.

Using formulae (2.15) and (2.18) we get, that near the critical curve the difference $T_{-} \equiv 1 / z_{h}(\tau-\ell)$ is finite and can be expressed in the form

$$
T_{-}=-\chi_{\kappa}(\phi),
$$

where function $\chi_{\kappa}$ is defined as

$$
\chi_{\kappa}(\phi) \equiv-\frac{1}{2} \log \mathcal{K}(\kappa, \phi) \mathcal{K}^{+}(\kappa, \phi)-\frac{1}{2 \kappa} \log \mathcal{K}^{-}(\kappa, \phi) .
$$

The second light cone variable $T_{+} \equiv \tau / z_{h}+\ell / z_{h}$ is singular near the critical line $T_{+} \sim$ $-\log \epsilon$. The function $\chi_{\kappa}(\phi)$ plays an important role in what follows, controlling the behavior of the light cone variable $T_{-}$. In the limit $\kappa \rightarrow 0$ we have $\chi_{\kappa}(\phi) \underset{\kappa \rightarrow 0}{\rightarrow} \chi_{0}(\phi)$, where

$$
\chi_{0}(\phi)=\cot \frac{\phi}{2}-1-\log \cot \frac{\phi}{2}
$$

is in accordance with [19]. The inverse function $\chi^{-1}$ can be expressed in terms of Lambert function $W$

$$
\phi=\chi_{0}^{-1}\left(T_{-}\right)=2 \operatorname{arccot}\left(-W_{-1}\left(-e^{T_{-}-1}\right)\right) .
$$


Now let us consider the entanglement entropy behavior near the critical line

$$
S-S_{\mathrm{eq}}=\log \frac{1}{\mathfrak{S}} \frac{\sinh \frac{\tau}{z_{h}}}{\sinh \frac{\ell}{z_{h}}} \approx \frac{\tau-\ell}{z_{h}}-2 \sinh \frac{\tau-\ell}{z_{h}} e^{-\frac{\tau+\ell}{z_{h}}}-\log \mathfrak{S}\left(\phi, \rho_{H}^{*}, \kappa\right) .
$$

Note, that $\mathfrak{S}(\phi, \rho, \kappa)$ is not singular on the critical line. Neglecting the exponentially small terms in (3.11) and introducing $\mathfrak{S}_{\kappa}(\phi)=\mathfrak{S}\left(\phi, \rho_{H}^{*}, \kappa\right)$ we get

$$
\Delta S \equiv S-S_{\mathrm{eq}} \approx T_{-}-\log \mathfrak{S}_{\kappa}(\phi) .
$$

Formula (3.8) gives the representation of $\phi$ in terms of $T_{-}$as $\phi=\chi_{\kappa}^{-1}\left(-T_{-}\right)$. Near the critical line the entanglement entropy has the wave spreading

$$
\Delta S \approx T_{-}-\log \mathfrak{S}_{\kappa}\left(\chi_{\kappa}^{-1}\left(-T_{-}\right)\right) .
$$

The dependence of entanglement entropy propagation only on $T_{-}$, i.e. realization of the memory loss regime, is based on the exponential suppression of $T_{+}$dependence, see (3.11), and dependence of $\mathfrak{S}$ only on $\phi$ near the critical line.

\subsubsection{Post-local-equilibration linear growth. Expansion near $\vartheta_{\kappa}$}

Above we established that the memory loss regime corresponds to the specific behavior of $\Delta S, \ell$ and $\tau$ in parametric space $\rho$ and $\phi$ near the critical curve resulting in dependence only on difference $\ell-\tau$. To pick out the specific behavior of entanglement corresponding to linear growth subregime let us analyse the behavior of $\ell$ on the critical curve. First let us consider $\ell_{-}$. The new feature of behavior of $\ell_{-}$on the critical curve for $\kappa>0$, is that it is singular near $\phi=\vartheta_{\kappa}$, where

$$
\vartheta_{\kappa}=\arccos \left(\frac{\sqrt{1+2 \kappa-3 \kappa^{2}}}{\sqrt{1+2 \kappa+\kappa^{2}}}\right) .
$$

When $\phi=\vartheta_{\kappa}+\delta$ we get

$$
\frac{\ell_{-}}{z_{h}} \approx-\frac{\log \delta}{2 \kappa}+\zeta_{\kappa}^{-}, \quad \zeta_{\kappa}^{-}=\frac{1}{2 \kappa} \log \left(\frac{4 \kappa\left(-2 \kappa^{2}+\kappa+1\right)}{(\kappa+1)^{2} \sqrt{(2-3 \kappa) \kappa+1}}\right) .
$$

Since time $\tau$ and $\ell_{+}$are not singular near this point we get

$$
-T_{-}=\chi_{\kappa}\left(\vartheta_{\kappa}+\delta\right)=-\frac{1}{2 \kappa} \log \delta+\zeta_{\kappa}, \quad \zeta_{\kappa}=\zeta_{\kappa}^{-}+\frac{1}{2} \log \frac{\kappa^{2}}{(1+\kappa)^{2}} .
$$

The function $\mathfrak{S}$ from equation (2.7) has the expansion near the critical line and at $\phi \rightarrow$ $\vartheta_{\kappa}+\delta$,

$$
\left.\mathfrak{S}_{\kappa}(\rho, s)\right|_{\rho=\rho_{c r}, \phi=\vartheta_{\kappa}+\delta}=\frac{2 \mathfrak{q}_{\kappa}}{\mathfrak{r}_{\kappa}} \delta^{1 / 2},
$$

where $\mathfrak{q}_{\kappa}$ depends on $\delta$ and $\mathfrak{r}_{\kappa}$ is some constant. We do not need to determine them explicitly. For completeness as an example at $\kappa=0.25$ we have $\mathfrak{q}_{0.25}=0.39 \delta$ and $\mathfrak{r}_{\kappa}$ is approximated by $\mathfrak{r}_{\kappa}=1.331-3(\kappa-0.334)^{2}$. 
Hence we have the expansion of $\Delta S$

$$
\Delta S \approx T_{-}-\log \frac{2 \mathfrak{q}_{\kappa}}{\mathfrak{r}_{\kappa}} e^{-\kappa\left(-T_{-}-\zeta_{\kappa}\right)}=(1-\kappa) T_{-}-\kappa \zeta_{\kappa}-\log \frac{2 \mathfrak{q}_{\kappa}}{\mathfrak{r}_{\kappa}} .
$$

Therefore, the linear coefficient is changed as compare to the case of the initial zero temperature, and it is

$$
\mathfrak{k}_{\kappa}=1-\kappa .
$$

The coefficient $\mathfrak{k}_{\kappa}$ is identified with the entanglement velocity $[19,55]$. The coefficient $\mathfrak{k}_{0}=1$ corresponds to $T_{i}=0$ and this is one of the fundamental facts about two-dimensional CFT. The fact, that initial temperature decreases this coefficient, can have two different explanations, see [55]. The first one is the presence of quasiparticles, streaming from the quench start with the speed less than CFT speed of sound. The second one is the consequence of particle multiple re-scattering and interactions. See [57, 59] for description of CFT in thermal state deformation by interaction. Now let us define $W(t)$ as a state of the half-space of our system at moment $t$. Then we define $w(t)$ as a boundary point of this half space. Finally define $X$ to be the null vicinity of point $w(t)$. The coefficient (3.19) also give rise to connection (see formula 2.9 in [55] and general discussion) with the mutual information $I(W(t), X)$

$$
\kappa \sim I(W(t), X) .
$$

This indicates presence of entanglement inside the effective emergent light cone between EPR pairs produced by quench.

\subsubsection{Saturation. Expansion near $\pi / 2$}

The equilibration process ends with the saturation regime. In [20,49] the dependence of entanglement entropy in this regime for $2 \mathrm{~d}$ holographic CFT for $T_{i}=0$ was found. We generalize this result on the case when initial temperature is not zero. Here we naturally consider the saturation as one of the limiting forms of memory loss regime, namely limit $T_{-} \rightarrow-0$ in (3.18). This corresponds to time scales near the final equilibration time $t_{s} \approx \ell$. In parametric space this regime corresponds to the values $\phi \rightarrow \pi / 2$.

Let us consider asymptotics of $\tau$ and $\ell=\ell_{-}+\ell_{+}$given by (2.18) and (2.15) when $\phi \rightarrow \pi / 2$. In this limit the asymptotic expansion for the combination $\ell-\tau$ takes the form

$$
\frac{\ell-\tau}{z_{h}}=\chi_{\kappa}(\phi) \approx \frac{z_{h} \delta^{2}}{2\left(1-\kappa^{2}\right)}+\frac{\left(z_{h}+2 \kappa^{2} z_{h}\right) \delta^{3}}{6\left(1-\kappa^{2}\right)^{3 / 2}}-\frac{\left(5 z_{h}+7 \kappa^{2} z_{h}\right) \delta^{4}}{24(1-\kappa)^{2}(1+\kappa)^{2}},
$$

where $\delta \approx \pi / 2-\phi$. The inverse function $\chi^{-1}$ is expressed in the form

$$
\begin{aligned}
\delta \approx & -\sqrt{2\left(1-\kappa^{2}\right)}\left(\frac{\ell-\tau}{z_{h}}\right)^{1 / 2}+\frac{\sqrt{1-\kappa^{2}}\left(1+2 \kappa^{2}\right)}{3} \frac{\ell-\tau}{z_{h}}- \\
& -\frac{\left(20 \kappa^{4}-\kappa^{2}-10\right) \sqrt{1-\kappa^{2}}}{18 \sqrt{2}}\left(\frac{\ell-\tau}{z_{h}}\right)^{3 / 2} .
\end{aligned}
$$

Finally, expanding $\log \mathfrak{S}$ near $\phi \rightarrow \pi / 2$

$$
\log \mathfrak{S} \approx \frac{\delta^{2}}{2\left(1-\kappa^{2}\right)}+\frac{\kappa^{2} \delta^{3}}{2\left(1-\kappa^{2}\right)^{3 / 2}}+\frac{\left(2+7 \kappa^{2}+3 \kappa^{4}\right) \delta^{4}}{24\left(1-\kappa^{2}\right)^{2}},
$$


we get

$$
\begin{aligned}
\Delta S & \approx-\mathfrak{f}_{\kappa}\left(\frac{\ell-\tau}{z_{h}}\right)^{3 / 2}-\mathfrak{n}_{\kappa}\left(\frac{\ell-\tau}{z_{h}}\right)^{2} \\
\mathfrak{f}_{\kappa} & =\frac{\left(\sqrt{2}\left(1-\kappa^{2}\right)\right)}{3}, \mathfrak{n}_{\kappa}=\frac{\left(1-\kappa^{2}\right)^{2}}{6},
\end{aligned}
$$

which generalizes the result obtained in $[19,49]$. We see that there is essential dependence of the coefficient $\mathfrak{f}_{\kappa}$ in front of scaling law on the initial temperature. Note, that at $\kappa=1$ all the expansion for saturation regime vanishes identically. The initial temperature dependence of the saturation regime involves only powers of $1-\kappa^{2}$.

\subsubsection{Late-time memory loss regime. Interpolation between $\phi=\vartheta_{\kappa}$ and $\pi / 2$}

There is an additional regime taking place on the timescale between linear growth and saturation interpolating between them. It is called [19] "late-time memory loss". In this regime the entanglement entropy depends only on time remaining till saturation. We outline some approximate formulae to describe this regime. As interpolation functions $\mathfrak{x}_{\kappa}(\phi)$ to $\chi_{\kappa}(\phi)$ on the interval $\left(\vartheta_{\kappa}, \pi / 2\right)$ we take

$$
\mathfrak{x}_{\kappa}=a_{\kappa}\left(\cot \frac{\phi-\vartheta_{\kappa}}{2 b_{\kappa}}-\cot \frac{\frac{\pi}{2}-\vartheta_{\kappa}}{2 b_{\kappa}}-\log \frac{\cot \frac{\phi-\vartheta_{\kappa}}{2 b_{\kappa}}}{\cot \frac{\frac{\pi}{2}-\vartheta_{\kappa}}{2 b_{\kappa}}}\right),
$$

where the numerical values of $a_{\kappa}$ and $b_{\kappa}$ are some numerical constants, for example $a_{0.1}=$ 0.15 and $b_{0.1}=2.5$. The inverse functions to $\mathfrak{x}_{\kappa}$ are given by

$$
\mathfrak{x}_{\kappa}^{-1} \equiv \phi=\vartheta_{\kappa}+2 b_{\kappa} \operatorname{arccot} W_{-1}\left(-e^{-\frac{\mathfrak{x}_{\kappa}}{a_{\kappa}}-\cot \phi_{\kappa, 0}} \cot \phi_{\kappa, 0}\right),
$$

where we define $\phi_{\kappa, 0}$ as

$$
\phi_{\kappa, 0} \equiv \frac{\frac{\pi}{2}-\vartheta_{\kappa}}{2 b_{\kappa}}
$$

Substituting $\phi_{\kappa, 0}$ in (3.18) we get

$$
\Delta S \approx T_{-}-\log \mathfrak{S}_{\kappa}\left(\vartheta_{\kappa}+2 b_{\kappa} \operatorname{arccot} W_{-1}\left(-e^{\frac{T}{a_{\kappa}}-\cot \phi_{\kappa, 0}} \cot \phi_{\kappa, 0}\right)\right) .
$$

\section{Conclusions and discussions}

In this paper using holographic approach, we have considered the evolution of entanglement entropy of single interval during equilibration after the global sharp quench of the initial thermal state at temperature $T_{i}$. This quench is followed by non-equilibrium heating up of the system to temperature $T_{f}$. We use Vaidya thin shell in the BTZ black hole background (so-called double-BTZ-Vaidya) as a holographic model of the process. The main purpose of this paper is to compare global quench process starting from thermal initial state with the one starting from the vacuum state. We have shown that the quench starting from thermal initial state shares all qualitative features of zero initial temperature case, but there is a quantitative dependence on $\kappa=T_{i} / T_{f}$. 
We have calculated the critical exponents corresponding to various regimes of entanglement entropy propagation. These exponents turn out to be independent on temperature. However, the coefficients $\mathfrak{f}_{\kappa}, \mathfrak{n}_{\kappa}, \mathfrak{k}_{\kappa}$ and $\mathfrak{g}_{\kappa}$ (see (3.3), (3.19) and (3.24)) corresponding to these exponents are $\kappa$-dependent. Namely, in section 3.2 we have derived the corrections to quadratic growth regime that occurs before local equilibrium is set. The pre-localequilibration stage in non-equilibrium heating is very similar to one during thermalization [20]. In our case the early growth time dependence is proportional to the difference of the energy densities

$$
\Delta S=2 \pi\left(\mathcal{E}_{f}-\mathcal{E}_{i}\right) \tau^{2}+\ldots,
$$

that is consistent with the initial evolution of HEE with $T_{i}=0$ [19, 20, 30-34]. For small interval size, the local equilibration scale $\xi \approx 1 / T_{f}$ is independent on the initial temperature.

In section 3.3, using the explicit formula for the evolution of the HEE, we have shown the existence of the memory loss regime in non-equilibrium heating. The HEE evolution in this regime is described by the function of one variable

$$
\Delta S(\ell, \tau) \approx \mathfrak{M}_{\kappa}(\ell-\tau)
$$

with an explicit dependence of the coefficient $\mathfrak{M}_{\kappa}$ on $\kappa$. The memory loss regime occurs long after the system has achieved local equilibration at scales of order $z_{h}$. However, we have found that details of memory loss explicitly depend on the initial temperature. Thus we find that only geometric data are lost when the system follows this regime, while some initial state details, like temperature, are resistant to be erased.

Similar to the thermalization [19], there are two special cases of the memory loss regime. In section 3.3.1 we have derived corrections to post-local-equilibration linear growth with large $\ell$, i.e. $z_{h} \ll \tau \ll \ell$. In this regime

$$
\mathfrak{M}_{\kappa}(\ell-\tau) \approx-\mathfrak{k}_{\kappa}(\ell-\tau)+\ldots,
$$

the scaling parameter $\mathfrak{k}_{\kappa}$ depends on $\kappa$ as

$$
\mathfrak{k}_{\kappa}=1-\kappa .
$$

This coefficient can be identified with the entanglement tsunami velocity. Following [55] there are two possible explanations for the reduction of this velocity. The first one may be related with the presence of quasiparticles, which travel with the speed less than the effective speed of the light. The second one, is due to multiple interactions between quasiparticles and interactions with thermal fluctuations. It is possible to interpret the decrease in the speed of a tsunami by the propagation of entanglement inside the light cone. The value on which this speed decreases allows an interpretation in terms of the mutual information.

Section 3.3.2 is devoted to calculation of the corrections to critical exponents at the saturation regime. This regime takes place when $\ell-\tau \ll z_{h}$, and

$$
\mathfrak{M}_{\kappa}(\ell-\tau) \approx-\mathfrak{f}_{\kappa}\left(\frac{\ell-\tau}{z_{h}}\right)^{3 / 2}-\mathfrak{n}_{\kappa}\left(\frac{\ell-\tau}{z_{h}}\right)^{2}+\ldots
$$


where the scaling parameter $\mathfrak{f}_{\kappa}$ depends on $\kappa$ as

$$
\mathfrak{f}_{\kappa}=\frac{\sqrt{2}}{3}\left(1-\kappa^{2}\right)
$$

and

$$
\mathfrak{n}_{\kappa}=\frac{1}{6}\left(1-\kappa^{2}\right)^{2}
$$

To summarize, we have shown that entanglement entropy evolution after the quench with the thermal initial state retained all regimes of the entanglement entropy evolution after the quench with vacuum initial state. All these evolution regimes, except saturation, are also present in the quench with a strongly inhomogeneous initial state [60] (for holographic description of single local quench see ).

It is worth to note, that in our model the equilibration time does not depend on the initial temperature. However, as we have seen, the speed of the entanglement propagation decreases with increasing of the initial temperature. Hence, one can conclude, that the reason for the entanglement speed reduction is the interaction of the quasiparticles stream with thermal fluctuations and this interaction is different at different stages of thermalization. In other words, one can say that the system responses to the reduction of the speed of entanglement propagation preserving full time of equilibration of the entanglement for the given interval.

For more complicated models, we expect that

$$
\Delta S(\ell, \tau) \approx \mathfrak{M}_{\kappa}(\ell-\mathfrak{v} \tau)
$$

where the form of $\mathfrak{M}_{\kappa}$ depends on the model, but the dependence on $(\ell-\mathfrak{v} \tau)$ with different value of $\mathfrak{v}$, will survive for more general initial states. In particular, for the linear growth regime we expect that the speed $\mathfrak{v}$, which characterizes properties of the equilibrium state, is solely determined by the black hole describing the final state. Equation (4.8) manifests itself the local nature of the entanglement propagation. s

It would be interesting to extend the results we have obtained to the HEE and the other nonlocal observables to a higher dimensional cases as well to study the heating process initiated by more general quenches, in particular quenches with inhomogeneous states (see for [58] $T_{i}=0$ case) or defined by various infalling shells. In particular these shells include massive infalling shells, charged shells, shells with angular momentum (corresponding thermalization process have been studied in [5, 16, 35-38, 51]). Thick shells infalling in the black hole background in higher dimensional cases have been already used to study numerically the holographic non-equilibrium heating [15]. When the thickness of the shell is less then typical sizes of intervals which we deal with, the evolution of the entanglement entropy for large intervals also shows the memory loss regime [20]. Also, it would be interesting to compare the results of this work and possible higher-dimensional generalizations with different results concerning equilibration of thermal states in holographic context including the numerical study of black hole with falling scalar field thin shell [46] and the holographic description of non-equilibrium thermal transport in two isolated quantum critical systems with different temperatures is found in [47, 48]. 


\section{Acknowledgments}

The authors are grateful to M. Khramtsov and M.Tikhanovskaya for useful discussions. This work is supported by the Russian Science Foundation (project 17-71-20154).

Open Access. This article is distributed under the terms of the Creative Commons Attribution License (CC-BY 4.0), which permits any use, distribution and reproduction in any medium, provided the original author(s) and source are credited.

\section{References}

[1] J.M. Maldacena, The Large-N limit of superconformal field theories and supergravity, Int. J. Theor. Phys. 38 (1999) 1113 [Adv. Theor. Math. Phys. 2 (1998) 231] [hep-th/9711200] [INSPIRE].

[2] S.S. Gubser, I.R. Klebanov and A.M. Polyakov, Gauge theory correlators from noncritical string theory, Phys. Lett. B 428 (1998) 105 [hep-th/9802109] [INSPIRE].

[3] E. Witten, Anti-de Sitter space and holography, Adv. Theor. Math. Phys. 2 (1998) 253 [hep-th/9802150] [INSPIRE].

[4] E. Witten, Anti-de Sitter space, thermal phase transition and confinement in gauge theories, Adv. Theor. Math. Phys. 2 (1998) 505 [hep-th/9803131] [INSPIRE].

[5] U.H. Danielsson, E. Keski-Vakkuri and M. Kruczenski, Black hole formation in AdS and thermalization on the boundary, JHEP 02 (2000) 039 [hep-th/9912209] [INSPIRE].

[6] J.M. Maldacena, Eternal black holes in anti-de Sitter, JHEP 04 (2003) 021 [hep-th/0106112] [INSPIRE].

[7] J. Casalderrey-Solana, H. Liu, D. Mateos, K. Rajagopal and U.A. Wiedemann, Gauge/String Duality, Hot QCD and Heavy Ion Collisions, in the book Gauge/String Duality, Hot QCD and Heavy Ion Collisions, Cambridge University Press, Cambridge U.K. (2014) [arXiv:1101.0618] [INSPIRE].

[8] I.Ya. Aref'eva, Holographic approach to quark-gluon plasma in heavy ion collisions, Phys. Usp. 57 (2014) 527.

[9] O. DeWolfe, S.S. Gubser, C. Rosen and D. Teaney, Heavy ions and string theory, Prog. Part. Nucl. Phys. 75 (2014) 86 [arXiv:1304.7794] [INSPIRE].

[10] S.A. Hartnoll, A. Lucas and S. Sachdev, Holographic quantum matter, arXiv:1612.07324 [INSPIRE].

[11] R. Easther, R. Flauger, P. McFadden and K. Skenderis, Constraining holographic inflation with WMAP, JCAP 09 (2011) 030 [arXiv:1104.2040] [INSPIRE].

[12] J. Abajo-Arrastia, J. Aparicio and E. Lopez, Holographic Evolution of Entanglement Entropy, JHEP 11 (2010) 149 [arXiv:1006.4090] [INSPIRE].

[13] V. Balasubramanian, A. Bernamonti, J. de Boer, N. Copland, B. Craps, E. Keski-Vakkuri et al., Holographic Thermalization, Phys. Rev. D 84 (2011) 026010 [arXiv:1103.2683] [INSPIRE].

[14] J. Aparicio and E. Lopez, Evolution of Two-Point Functions from Holography, JHEP 12 (2011) 082 [arXiv:1109.3571] [INSPIRE]. 
[15] I.Ya. Aref'eva and I.V. Volovich, Holographic thermalization, Theor. Math. Phys. 174 (2013) 186 [Teor. Mat. Fiz. 174 (2013) 216] [arXiv:1211.6041].

[16] I.Ya. Aref'eva, A. Bagrov and A.S. Koshelev, Holographic Thermalization from Kerr-AdS, JHEP 07 (2013) 170 [arXiv: 1305.3267] [INSPIRE].

[17] Y.-Z. Li, S.-F. Wu, Y.-Q. Wang and G.-H. Yang, Linear growth of entanglement entropy in holographic thermalization captured by horizon interiors and mutual information, JHEP 09 (2013) 057 [arXiv: 1306. 0210] [INSPIRE].

[18] T. Hartman and J. Maldacena, Time Evolution of Entanglement Entropy from Black Hole Interiors, JHEP 05 (2013) 014 [arXiv: 1303.1080] [INSPIRE].

[19] H. Liu and S.J. Suh, Entanglement Tsunami: Universal Scaling in Holographic Thermalization, Phys. Rev. Lett. 112 (2014) 011601 [arXiv:1305.7244] [INSPIRE].

[20] H. Liu and S.J. Suh, Entanglement growth during thermalization in holographic systems, Phys. Rev. D 89 (2014) 066012 [arXiv:1311.1200] [INSPIRE].

[21] S. Leichenauer and M. Moosa, Entanglement Tsunami in (1+1)-Dimensions, Phys. Rev. D 92 (2015) 126004 [arXiv: 1505.04225] [INSPIRE].

[22] S.H. Shenker and D. Stanford, Black holes and the butterfly effect, JHEP 03 (2014) 067 [arXiv: 1306.0622] [INSPIRE].

[23] D.S. Ageev and I.Ya. Aref'eva, Waking and scrambling in holographic heating up, Teor. Mat. Fiz. 193 (2017) 146 [arXiv:1701.07280] [InSPIRE].

[24] V.E. Hubeny and H. Maxfield, Holographic probes of collapsing black holes, JHEP 03 (2014) 097 [arXiv: 1312.6887] [INSPIRE].

[25] T. Albash and C.V. Johnson, Evolution of Holographic Entanglement Entropy after Thermal and Electromagnetic Quenches, New J. Phys. 13 (2011) 045017 [arXiv:1008.3027] [INSPIRE].

[26] V. Balasubramanian, A. Bernamonti, J. de Boer, N. Copland, B. Craps, E. Keski-Vakkuri et al., Thermalization of Strongly Coupled Field Theories, Phys. Rev. Lett. 106 (2011) 191601 [arXiv: 1012 .4753] [INSPIRE].

[27] S. Ryu and T. Takayanagi, Holographic derivation of entanglement entropy from AdS/CFT, Phys. Rev. Lett. 96 (2006) 181602 [hep-th/0603001] [INSPIRE].

[28] V.E. Hubeny, M. Rangamani and T. Takayanagi, A Covariant holographic entanglement entropy proposal, JHEP 07 (2007) 062 [arXiv: 0705.0016] [INSPIRE].

[29] J. Aparicio and E. Lopez, Evolution of Two-Point Functions from Holography, JHEP 12 (2011) 082 [arXiv:1109.3571] [INSPIRE].

[30] D. Allahbakhshi, M. Alishahiha and A. Naseh, Entanglement Thermodynamics, JHEP 08 (2013) 102 [arXiv:1305.2728] [INSPIRE].

[31] J. Bhattacharya, M. Nozaki, T. Takayanagi and T. Ugajin, Thermodynamical Property of Entanglement Entropy for Excited States, Phys. Rev. Lett. 110 (2013) 091602 [arXiv: 1212.1164] [INSPIRE].

[32] M. Nozaki, T. Numasawa, A. Prudenziati and T. Takayanagi, Dynamics of Entanglement Entropy from Einstein Equation, Phys. Rev. D 88 (2013) 026012 [arXiv:1304.7100] [INSPIRE]. 
[33] D.D. Blanco, H. Casini, L.-Y. Hung and R.C. Myers, Relative Entropy and Holography, JHEP 08 (2013) 060 [arXiv: 1305.3182] [INSPIRE].

[34] G. Wong, I. Klich, L.A. Pando Zayas and D. Vaman, Entanglement Temperature and Entanglement Entropy of Excited States, JHEP 12 (2013) 020 [arXiv:1305.3291] [INSPIRE].

[35] U.H. Danielsson, E. Keski-Vakkuri and M. Kruczenski, Spherically collapsing matter in AdS, holography and shellons, Nucl. Phys. B 563 (1999) 279 [hep-th/9905227] [INSPIRE].

[36] J. Erdmenger and S. Lin, Thermalization from gauge/gravity duality: Evolution of singularities in unequal time correlators, JHEP 10 (2012) 028 [arXiv:1205.6873] [INSPIRE].

[37] D. Galante and M. Schvellinger, Thermalization with a chemical potential from AdS spaces, JHEP 07 (2012) 096 [arXiv: 1205.1548] [INSPIRE].

[38] E. Caceres and A. Kundu, Holographic Thermalization with Chemical Potential, JHEP 09 (2012) 055 [arXiv: 1205.2354] [INSPIRE].

[39] W. Baron, D. Galante and M. Schvellinger, Dynamics of holographic thermalization, JHEP 03 (2013) 070 [arXiv: 1212.5234] [INSPIRE].

[40] V. Keranen, E. Keski-Vakkuri and L. Thorlacius, Thermalization and entanglement following a non-relativistic holographic quench, Phys. Rev. D 85 (2012) 026005 [arXiv:1110.5035] [INSPIRE].

[41] M. Alishahiha, A. Faraji Astaneh and M.R. Mohammadi Mozaffar, Thermalization in backgrounds with hyperscaling violating factor, Phys. Rev. D 90 (2014) 046004 [arXiv: 1401.2807] [INSPIRE].

[42] P. Fonda, L. Franti, V. Keränen, E. Keski-Vakkuri, L. Thorlacius and E. Tonni, Holographic thermalization with Lifshitz scaling and hyperscaling violation, JHEP 08 (2014) 051 [arXiv: 1401.6088] [INSPIRE].

[43] I.Ya. Aref'eva, Formation time of quark-gluon plasma in heavy-ion collisions in the holographic shock wave model, Teor. Mat. Fiz. 184 (2015) 398 [arXiv:1503.02185] [INSPIRE].

[44] I.Ya. Aref'eva, A.A. Golubtsova and E. Gourgoulhon, Analytic black branes in Lifshitz-like backgrounds and thermalization, JHEP 09 (2016) 142 [arXiv:1601.06046] [INSPIRE].

[45] I.Ya. Aref'eva and I. Volovich, Holographic Photosynthesis, arXiv:1603.09107 [InSPIRE].

[46] K. Landsteiner, E. Lopez and G. Milans del Bosch, Quenching the Chiral Magnetic Effect via the Gravitational Anomaly and Holography, Phys. Rev. Lett. 120 (2018) 071602 [arXiv:1709.08384] [INSPIRE].

[47] M.J. Bhaseen, B. Doyon, A. Lucas and K. Schalm, Far from equilibrium energy flow in quantum critical systems, Nature Phys. 11 (2015) 5 [arXiv:1311.3655] [INSPIRE].

[48] J. Erdmenger, D. Fernandez, M. Flory, E. Megias, A.-K. Straub and P. Witkowski, Time evolution of entanglement for holographic steady state formation, JHEP 10 (2017) 034 [arXiv: 1705. 04696] [INSPIRE].

[49] V.E. Hubeny, M. Rangamani and E. Tonni, Thermalization of Causal Holographic Information, JHEP 05 (2013) 136 [arXiv: 1302.0853] [INSPIRE].

[50] V. Ziogas, Holographic mutual information in global Vaidya-BTZ spacetime, JHEP 09 (2015) 114 [arXiv: 1507.00306] [INSPIRE]. 
[51] S. Kundu and J.F. Pedraza, Spread of entanglement for small subsystems in holographic CFTs, Phys. Rev. D 95 (2017) 086008 [arXiv:1602.05934] [InSPIRE].

[52] P. Calabrese and J.L. Cardy, Evolution of entanglement entropy in one-dimensional systems, J. Stat. Mech. 0504 (2005) P04010 [cond-mat/0503393] [INSPIRE].

[53] P. Calabrese and J.L. Cardy, Time-dependence of correlation functions following a quantum quench, Phys. Rev. Lett. 96 (2006) 136801 [cond-mat/0601225] [INSPIRE].

[54] S. Sotiriadis, P. Calabrese and J. Cardy, Quantum Quench from a Thermal Initial State, Europhys. Lett. 87 (2009) 20002 [arXiv:0903.0895].

[55] H. Casini, H. Liu and M. Mezei, Spread of entanglement and causality, JHEP 07 (2016) 077 [arXiv: 1509.05044] [INSPIRE].

[56] M. Mezei, On entanglement spreading from holography, JHEP 05 (2017) 064 [arXiv: 1612.00082] [INSPIRE].

[57] J. Cardy, Quantum Quenches to a Critical Point in One Dimension: some further results, J. Stat. Mech. 1602 (2016) 023103 [arXiv: 1507.07266] [INSPIRE].

[58] V. Balasubramanian, A. Bernamonti, J. de Boer, B. Craps, L. Franti, F. Galli et al., Inhomogeneous holographic thermalization, JHEP 10 (2013) 082 [arXiv:1307.7086] [INSPIRE].

[59] L. McGough, M. Mezei and H. Verlinde, Moving the CFT into the bulk with TT , arXiv: 1611.03470 [INSPIRE].

[60] I.Ya. Aref'eva, M.A. Khramtsov and M.D. Tikhanovskaya, Thermalization after holographic bilocal quench, JHEP 09 (2017) 115 [arXiv:1706.07390] [INSPIRE].

[61] M. Nozaki, T. Numasawa and T. Takayanagi, Holographic Local Quenches and Entanglement Density, JHEP 05 (2013) 080 [arXiv: 1302.5703] [INSPIRE]. 